\title{
"Proteomic markers of DNA repair and PI3K pathway activation predict response to the PARP inhibitor BMN 673 in small cell lung cancer" - Letter
}

\author{
Robert JG Cardnell and Lauren A. Byers
}

In a letter to the Editor regarding our recent publication (1), Haifeng Qiu suggests further clarification is needed regarding the relationship between sensitivity to BMN 673 and dysregulated PI3K/mTOR signaling, given the potential association between PTEN loss and PARP inhibitor sensitivity in other cancer types.

While we agree that PTEN inactivation is common in human malignancies and may be associated with sensitivity to PARP inhibitors in some cancers, PTEN loss is only one of several possible mechanisms by which the PI3K/mTOR pathway can be activated, each of which may have different implications for drug sensitivity. For SCLC, this distinction was illustrated in a recent publication (2) that showed activity of a selective oral PI3K inhibitor, PF-4989216, in small cell lung cancer (SCLC) pre-clinical models harboring activating PIK3CA mutations, but not PTEN loss.

Genomic analysis by Peifer et al (3) indicates that PTEN loss occurs in a minority SCLC tumors (10\%). Of the cell lines used in our analysis, only one - NCI-H2081 - carries a PTEN mutation and this cell line was not included in the proteomic analysis. The H2081 cell line fell in the middle of the range of sensitivities to $\mathrm{BMN} 673\left(6.3 \mathrm{nM} \mathrm{IC}_{50}\right.$, range 1.7$15 \mathrm{nM}$ ) suggesting that PTEN deficiency was not responsible for the observed sensitivity of our SCLC cell lines to BMN 673, nor was it the cause of greater PI3k/mTOR pathway activation observed in the more resistant lines.

As described in Dr. Qiu's letter, an association between PTEN deficiency and PARP inhibitor sensitivity has been described in other cancers, including breast, prostate, and endometrial cancers. However, even among cancers with PTEN loss, there are likely to be additional factors that impact sensitivity, as suggested by a recent study that showed sensitivity of tumors with PTEN loss was dependent on levels of circulating estrogen (4). Furthermore, the potential benefit of dual PARP-PI3K pathway inhibition has also been suggested in breast cancer based on preclinical models of triple negative and BRCA mutated breast cancers (5) and is being explored in an ongoing clinical trial.

Correspondence to: Lauren A. Byers M.D., Department of Thoracic/Head and Neck medical Oncology, UT MD Anderson Cancer Center, 1515 Holcombe Blvd, Unit 0432, Houston TX, 77030. lbyers@ mdanderson.org. Phone: 713-792-6363 Fax: 713-792-1220. Conflicts:

LAB is a Principal Investigator on a Phase I, first in human, single-arm, open-label study of once a day, orally administered BMN 673 (PARP inhibitor) in patients with advanced or recurrent solid tumors. 
In conclusion, we agree with Dr. Qiu that the connection between PARP inhibitors and the PI3K/mTOR pathway warrants further exploration. However, our data indicates that the association between baseline PI3K/mTOR pathway activity and in vitro activity of BMN673 is independent of PTEN and suggests a distinct mechanism of PARP inhibitor activity in SCLC.

\section{References}

1. Cardnell RJ, Feng Y, Diao L, Fan YH, Masrorpour F, Wang J, et al. Proteomic Markers of DNA Repair and PI3K Pathway Activation Predict Response to the PARP Inhibitor BMN 673 in Small Cell Lung Cancer. Clinical cancer research: an official journal of the American Association for Cancer Research. 2013; 19:6322-8. [PubMed: 24077350]

2. Walls M, Baxi SM, Mehta PP, Liu KK, Zhu J, Estrella H, et al. Targeting Small Cell Lung Cancer Harboring PIK3CA Mutation with a Selective Oral PI3K Inhibitor PF-4989216. Clinical cancer research: an official journal of the American Association for Cancer Research. 2013

3. Peifer M, Fernandez-Cuesta L, Sos ML, George J, Seidel D, Kasper LH, et al. Integrative genome analyses identify key somatic driver mutations of small-cell lung cancer. Nature genetics. 2012; 44:1104-10. [PubMed: 22941188]

4. Janzen DM, Paik DY, Rosales MA, Yep B, Cheng D, Witte ON, et al. Low Levels of Circulating Estrogen Sensitize PTEN-Null Endometrial Tumors to PARP Inhibition In Vivo. Molecular cancer therapeutics. 2013; 12:2917-28. [PubMed: 24222661]

5. Alderton GK. Therapeutics: PI3K-PARP combination. Nature reviews Cancer. 2012; 12:658. 